\title{
Microcontroller based high voltage, high speed trigger control circuit for SMARTEX-C
}

\author{
MINSHA SHAH, HITESH MANDALIYA, LAVKESH LACHHVANI, MANU BAJPAI, \\ RACHANA RAJPAL \\ Electronics and Instrumentation Division \\ Institute for Plasma Research \\ Near Indira Bridge, Bhat Village, Gandhinagar, Gujarat \\ INDIA
}

\begin{abstract}
Microcontroller based trigger control circuit for fast pulsing of electrode potentials on wide range of time scales has been designed, installed, and tested for electron plasma experiments which are carried out in partial toroidal trap SMall Aspect Ratio Toroidal Electron plasma EXperiment in C - shaped geometry (SMARTEX - C), a device to create and confine non-neutral plasma (electron plasma). The sequence of trap operation is inject-hold-dump for which electrodes need to be pulsed with applied voltages at a high switching speed of few nanoseconds. Also this sequence of operation needs to be controlled over a very wide range of time scales from few microseconds to few seconds. As the available COTS (Commercial-Off-The-Shelf) high voltage DC power supplies generally do not provide this feature of fast switching at nanosecond time scale, MOSFET based circuit is developed which provides fast switching in the range of $20-100$ nanoseconds of high voltages $(200 \mathrm{Vdc}-500 \mathrm{Vdc})$ of multiple electrodes. The timing pulse widths of these trigger pulses are controlled using a microcontroller-based circuit. This experimental set-up also requires the triggering of a high current dc power supply used for an Electro-magnet (Toroidal Field Coil) to generate a toroidal magnetic field, at the start of this experiment. For this purpose, a Silicon Controlled Rectifier (SCR) based circuit is used. The gate pulse to trigger the SCR circuit is also generated from this microcontroller-based circuit. National Instrument's LabVIEW software based Graphical User Interface (GUI) is developed for triggering the SCR and electrodes with a programmable time period through the serial link.
\end{abstract}

Key-Words: - Microcontroller, B-field, Trigger, Injector grid, Collector grid, GUI, Electron Plasma.

Received: January 3, 2021. Revised: August 7, 2021. Accepted: September 1, 2021. Published: September 18, 2021.

\section{Introduction}

Electron plasma experiments are being carried out at the Institute for Plasma Research in SMARTEX - C (partial) torus [1]. Experiments are carried out in Ultra-High Vacuum environment $\leq 5 \times 10^{-9}$ mbar. Trap is operated 'a la' cylindrical trap in an 'injecthold-dump' scheme [2]. Electrons are injected thermionically from tungsten filament which is biased at about $-330 \mathrm{Vdc}$ and heated with a steady current $\sim 18 \mathrm{~A}$. An injector grid is mounted in front of this filament and biased at a lower voltage at about $-370 \mathrm{Vdc}$ with respect to the tungsten filament to prevent the electron-injection into the trapping region. Another grid called, collector grid is also maintained at about $-370 \mathrm{Vdc}$ and mounted behind the filament. The injector grid and collector grid are placed toroidally $315^{\circ}$ apart from each other and separated by a stainless-steel plate which is electrically floating, forming a trap region between the two. The arrangement of the electrodes in SMARTEX-C is shown in Fig.1. Trap dimensions are also shown in the Fig.1.

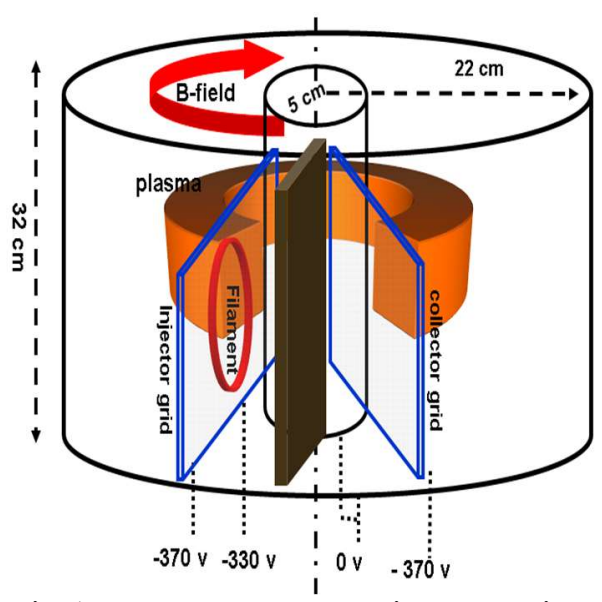

Fig.1 SMARTEX-C Device Overview

\section{Requirement Specifications}

Electron plasma in the trap is produced in sequence of 'Inject - hold - dump' as follows: At the start of the experiment, a preset toroidal magnetic field is created by passing the current in the toroidal field coil. The trigger circuit sends the trigger pulse to TF 
coil (B-field) power supply. Once the B-field reaches its flat-top, the injector grid is pulsed, i.e. brought to $0 \mathrm{~V}$ momentarily (say for 60 microseconds) to inject the electrons in the trap and switched back to $-370 \mathrm{~V}$ to stop further injection. Thus, the Plasma is trapped between injector grid and collector grid for some time. The time for which the plasma is trapped is termed as 'hold time', which can be defined as the time between falling edge of injector pulse and rising edge of collector pulse. To measure the total trapped charge at any given instant, after a preset hold time, the collector grid is switched to $0 \mathrm{~V}$ (say for 60 microseconds) in order to measure the trapped charge [3]. For all of this microsecond time intervals and controllable pulse widths micro-controller based trigger circuit is designed. The timing sequence of pulsing electrodes in Inject-hold-dump cycle is shown in Fig.2

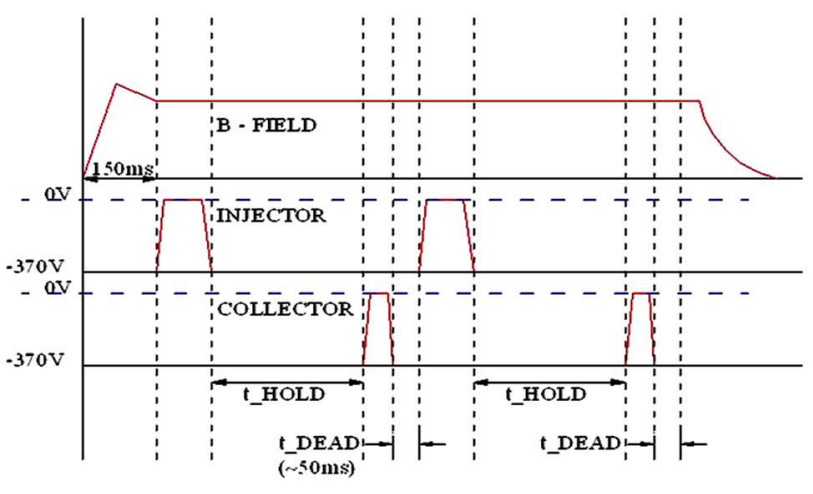

Fig.2 An indicative timing sequence of magnetic field and multiple injector \& collector grid pulses (two such pulses are shown as an example)

\section{Hardware Description}

A microcontroller based trigger circuit [4] has been developed in-house for the control and pulsing of high voltage applied to electrodes and to trigger the SCR for magnetic field power supply. In the first phase of this experiment it is required to have pulses with programmable time delays of 60 microseconds to 1 second with the step resolution of 60 microseconds. As in this application any kind of pulse width modulation is not needed, instead a precise programmable delay is needed to apply a single pulse to MOSFET; we have started implementing this application using low cost AT89C52 which is easily available in our stock. The assembly language is perfect to generate software delay and the programming tool needed for this MCU is also available with us. Also it is required to have fast switching of high voltage for electrodes; because due to the slower rise time the charge would be lost to the vessel boundaries which will reduce the self-electric field and give incorrect information of the trapped charge. In order to achieve fast switching of high voltage applied on injector and collector grids, MOSFETs are used as switching device. Also it is required to on/off injector and collector several times as per the number of triggers until the falling edge of magnetic field which we call as plasma shot. However the pulse widths for injector and collector will remain fixed for each trigger for the entire shot duration. The complete hardware consists of AT89C52, Darlington Transistor array IC, MOSFET drivers, MOSFETs, Optical Isolators, Isolated RS232 line transceiver. The complete hardware architecture is shown by a block diagram in Fig. 3 .

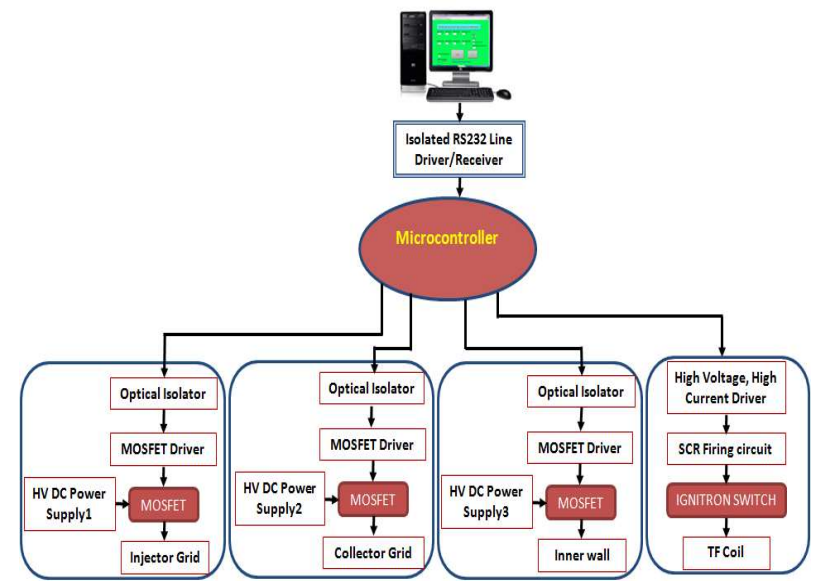

Fig.3 Electronics Hardware Block Diagram

An ignitron is used as a switch to discharge the toroidal field (TF) coil. This switch is operated by thyristor (SCR) which in turn is controlled by microcontroller. A fixed pulse of 250 microseconds is generated by microcontroller to fire the gate of SCR through pulse transformer to isolate the trigger circuit ground \& the TF magnetic field circuit ground. To drive the pulse transformer, IC ULN2003 is used. The scheme is shown in Fig.4

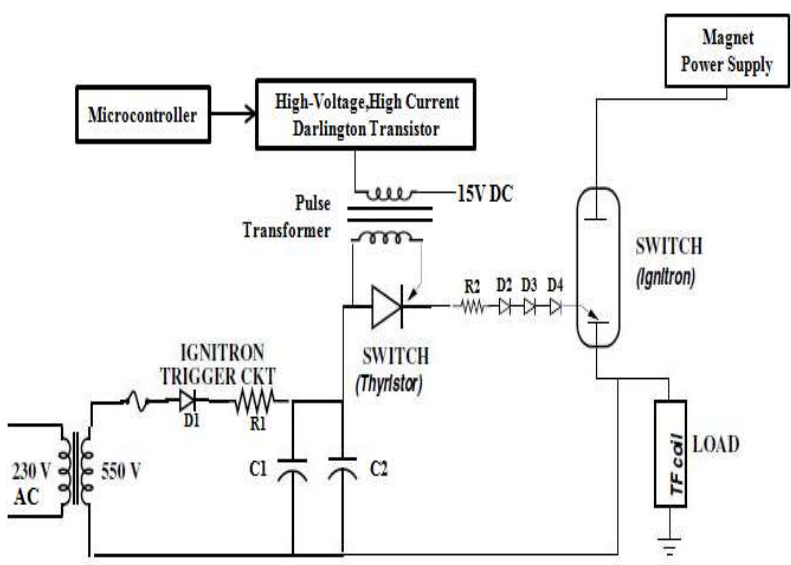

Fig.4 Magnetic field power-supply trigger circuit 
The microcontroller generates individual trigger pulses for injector, collector, inner wall and SCR. These trigger pulses are optically isolated using HCPL2631 for safety. These isolated trigger pulses drives MOSFET driver which in turn triggers MOSFET. IRFBC30 MOSFETs are used as a switch to pulse the power applied to electrodes. To quickly charge the gate capacitance to power the MOSFET, IR2110 MOSFET drivers are used. Separate MOSFET drivers and MOSFETS are used for injector, collector and inner wall. The MOSFET is biased at its source at around $370 \mathrm{~V}$. Transformer based floating power supplies are designed for each MOSFET driver. The MOSFET based power circuit \& DC power supply arrangement is shown in Fig.5

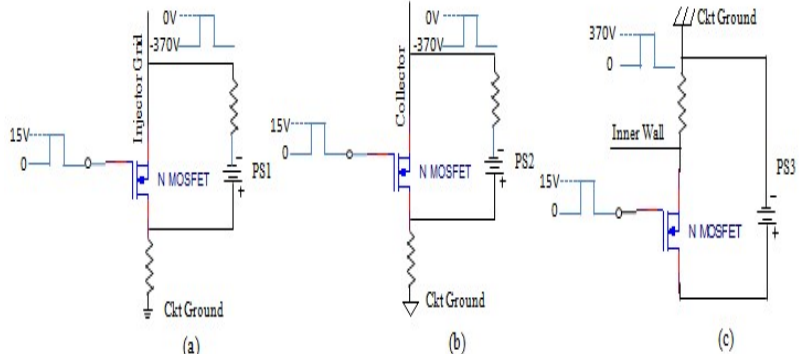

Fig.5 MOSFET Configurations for (a) Injector grid (b) Collector grid and (c) Inner wall.

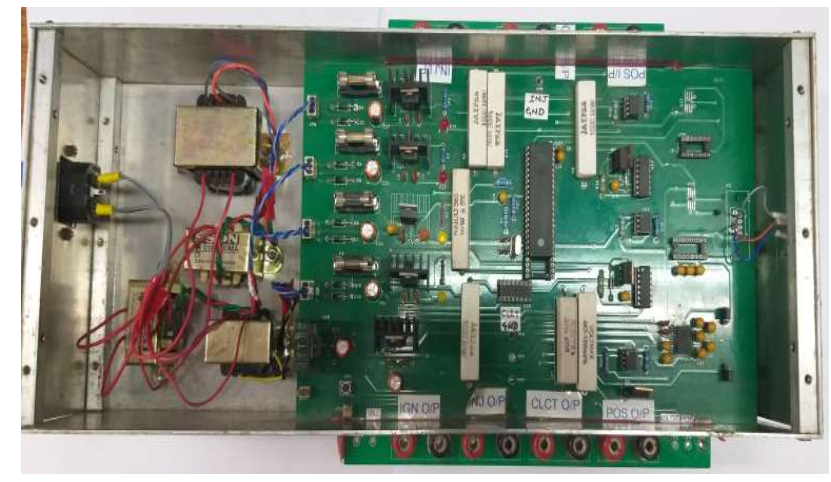

Fig.6 Trigger Circuit

\section{Software Description}

\subsection{Graphical User Interface (GUI)}

The GUI is developed in National Instrument's LabVIEW software to remotely control various parameters through host PC. Once the GUI application runs, the Trigger button gets enabled and the user can enter the parameters such as injector pulse On/Off time, collector pulse On/Off time and positive pulse On/Off time through GUI. The timing can be changed with the resolution of 60 microseconds. Also number of triggers between a plasma shot can be set through GUI. After setting all the control parameters, trigger button is pressed which sends all these parameters through a serial link to the hardware. Fig.7 shows the front panel of GUI and Fig.8 shows logic flow chart of GUI.

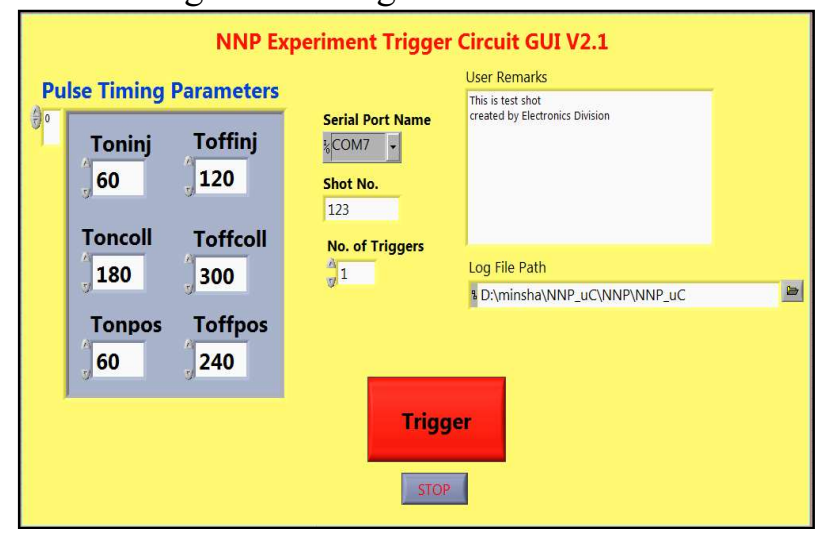

Fig.7 Graphical User Interface (GUI)

\subsubsection{Logic flow chart}

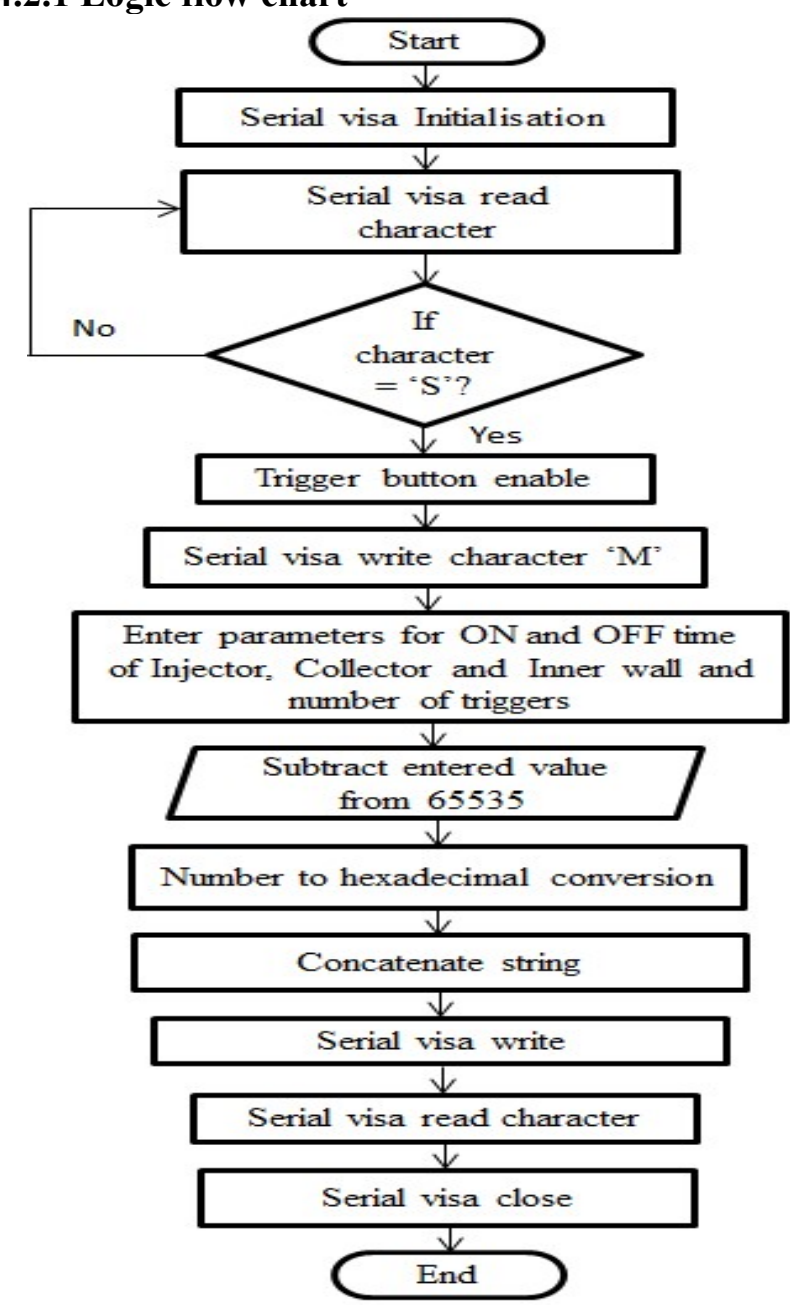

Fig.8 GUI Logic Flow chart

\subsection{Microcontroller logic implementation}

The logic is implemented using Keil uVision IDE embedded software toolset. The pulse width and delay between pulses for various grids are implemented using soft delay loops and comparing with the preset timing value which are stored in 
different variables. The logic flow chart is shown in Fig. 9

\subsubsection{Logic flow chart}

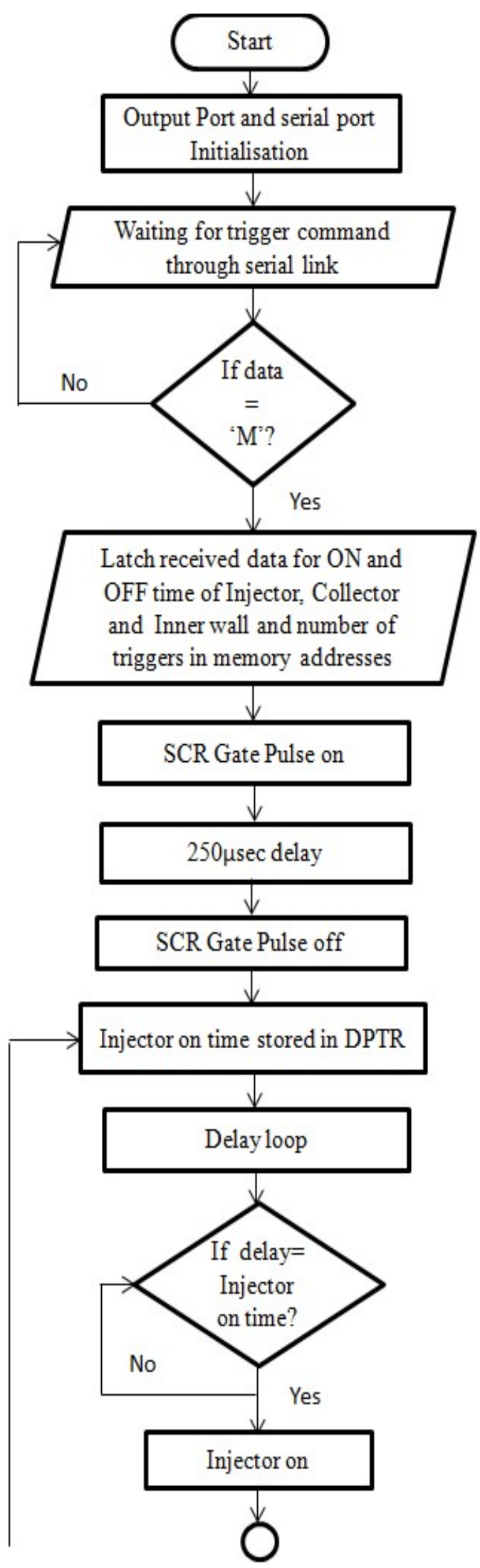

Fig.9 Microcontroller Logic Flow chart

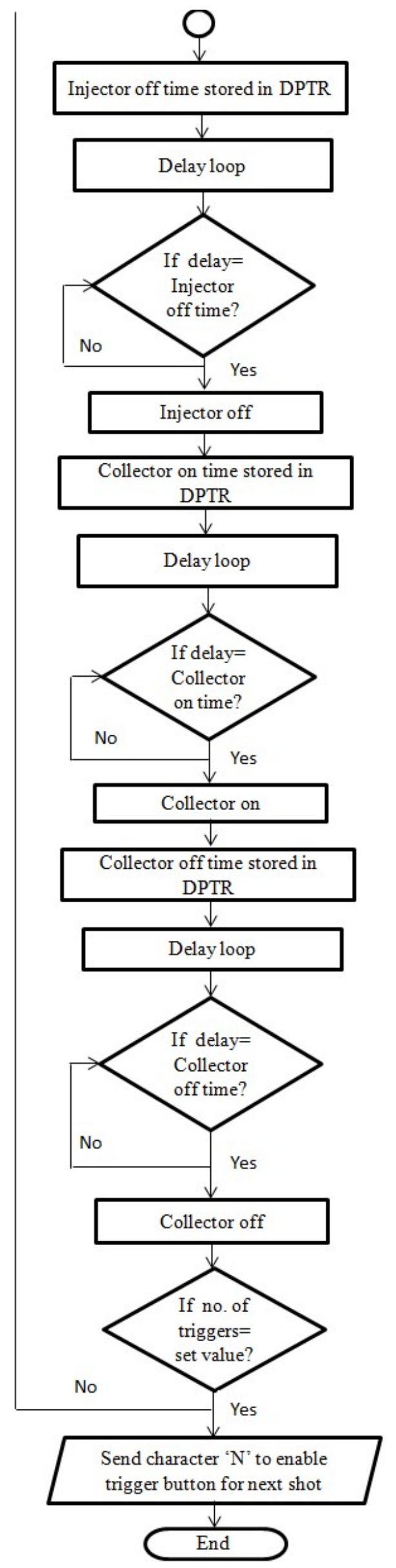




\section{Results \& Conclusion}

This section discusses about the experimental results obtained using the trigger control box.

Voltage trigger pulses without load condition for injector and collector grid are shown in Fig.10 Hold time in this test shot is typically 60 microseconds. Injector pulse width has been kept as 60 microseconds and collector grid pulse width has been set as 120 microseconds. Width of injector pulse width and collector pulse width has been denoted with $\tau$ inj and $\tau$ coll in Fig. 10 and delay between these two pulse has been denoted as $\tau$ thold.

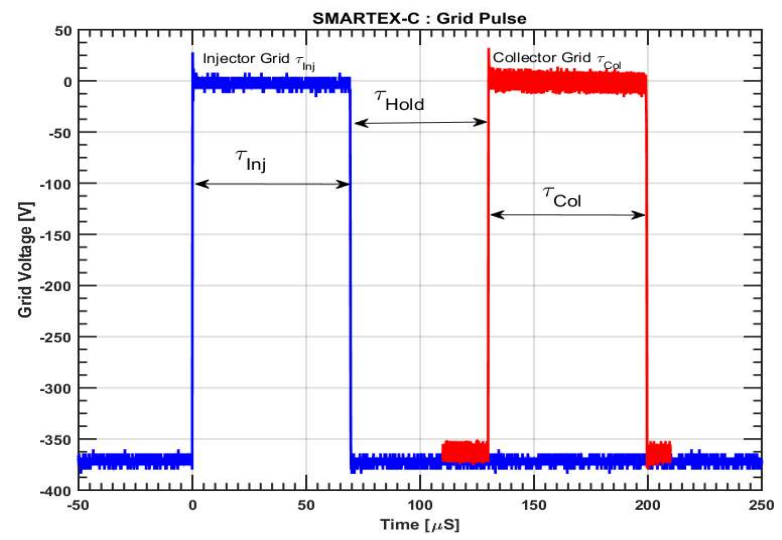

Fig.10 Output pulses from trigger circuit without load for injector grid and collector grid

Rise time of both the pulses with no-load and with load condition is shown in Fig.11. In 'no-load' condition, the circuit offers a rise time of 60-70 ns. When the circuit is loaded with the grids, the rise time of the pulses reaches $80-100 \mathrm{~ns}$ which is much faster than the observed $\mathrm{E} \times \mathrm{B}$ time scales $\sim 20 \mu$ s as desired. Jitter measurement has been obtained by taking 10 repetitive shots consequently in CRO in jitter measurement mode and it has been found 40 nanoseconds.

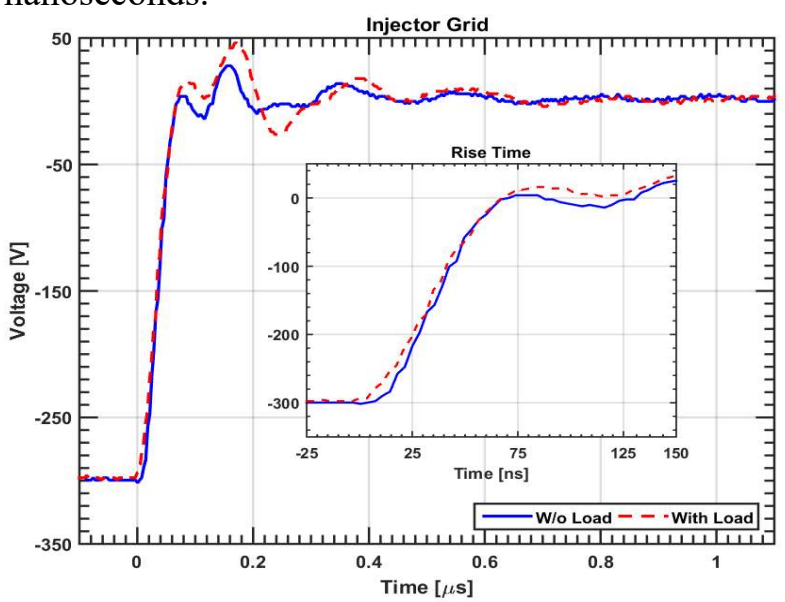

Fig.11 Rise time of injector grid pulse with load (Injector grid) and without load are plotted. Inset shows the zoom of the pulse showing rise time.
Electron plasmas in cylindrical traps in uniform magnetic field are routinely confined for very long times at thermal equilibrium, whereas toroidal electron plasmas lack the excellent confinement properties. This experimental setup attempts to investigate the effect of tight aspect ratio on the confinement properties of electron plasma and study the transport of charged particles in presence of strong non-uniform toroidal magnetic field.

Electron plasma in SMARTEX-C using microcontroller based trigger box has been produced and results of the experiments are shown in Fig. 12. The plasma shot begins with the triggering of Bfield. As soon as it reaches flat-top, the injector grid is pulsed to zero volts (red line) to inject the electrons and at the end of the shot collector grid is pulsed to dump the charges trapped in the vessel at that instant of time. Curve in blue shows the capacitive probe oscillations (due to destabilized diocotron oscillations) till the collector/dump pulse. Inset plot shows that as soon as the collector grid is pulsed, diocotron oscillations disappear and plasma is destroyed. Fig. 13 shows experimental results with different hold time.

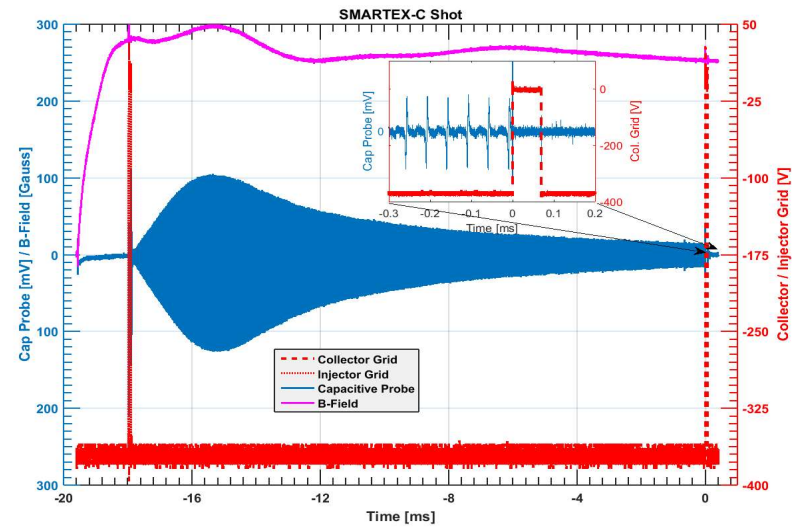

Fig.12 Typical plasma shot taken with microcontroller based trigger box. B-field pulse, Injector grid pulse, capacitive probe diagnostics data, collector grid pulse are shown indicating, Inject Hold and Dump phase of the experiment.

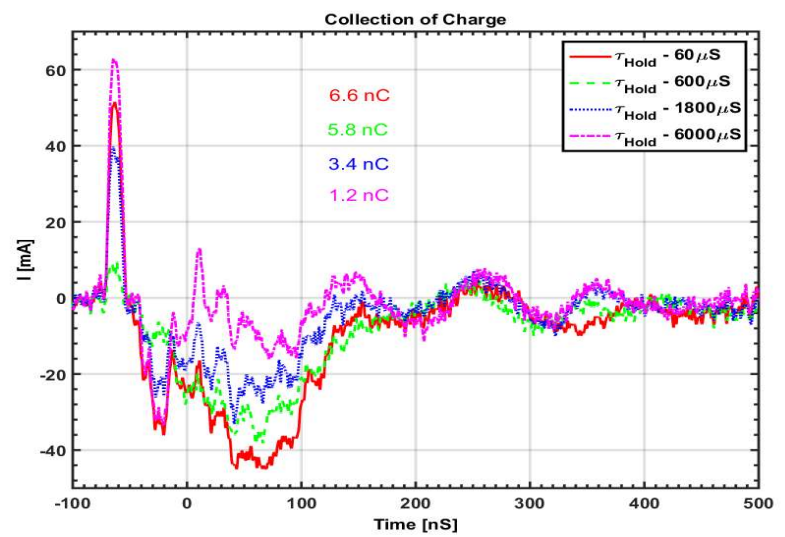

Fig. 13 Charge collection with different hold time 


\section{Future Scope}

The microcontroller based trigger circuit has been used several times to create and characterize the plasma. It has been found that due to less jitter in the circuit, plasma is highly reproducible. Though less time resolution limits the characterization of the plasma at this interval only and restricts the investigation of plasma dynamics occurring in this short interval and hence we foresee the requirement of a control circuit having time resolution around $1 \mu \mathrm{s}$ or better. Also the new power supply to charge the TF coil, replacing the SCR and ignitron switch, is procured which needs to generate the pulse of maximum $10 \mathrm{sec}$ width. Also it is required to provide multiple trigger facility of injector and collector with same or variable hold time for each trigger until magnetic field power supply is switched off. In future it may be required in some experiment to bring the collector grid to zero potential depending upon the number of zero cross detection of diocotron oscillations rather than on time scale basis. So in future design option to detect zero cross detection of diocotron oscillations and collector grid switching option, either time base or zero cross detection, also needs to be implemented. In future experiment it may also be required to disable collector grid pulse in case if we do not want to dump the plasma. For the next phase of experiments which demands more complex timing pattern as mentioned above, advance controllers or FPGA based trigger circuit will be designed and developed in future. Modern FPGAs can be easily configured for System on chip solutions. This facilitates very little use of external components to implement a whole system in a single chip. As FPGAs are fast and allow concurrent processing of time critical functions it is possible to generate independent trigger pulses for 1) magnetic field power supply 2) injector grid 3) collector grid and 4) inner wall with variable pulse width of few microseconds to tens of seconds with the resolution of $1 \mu \mathrm{s}$.
References:

[1] S. Pahari, H. S. Ramachandran, and P. I. John, "Electron plasmas: Confinement and mode structure in a small aspect ratio toroidal experiment," Phys. Plasmas, vol. 13, no. 9, p. 092111, 2006.

[2] J. H. Malmberg and J. S. deGrassie, "Properties of Nonneutral Plasma," Phys. Rev. Lett., vol. 35, no. 9, pp. 577-580, Sep. 1975.

[3] S. Pahari, L. Lachhvani, M. Bajpai, K. Rathod, Y. Yeole, and P. K. Chattopadhyay, "Design, development, and results from a chargecollector diagnostic for a toroidal electron plasma experiment," Review of Scientific Instruments, vol. 86, no. 8, p. 083504, Aug. 2015.

[4] Praveenlal Edappala, Minsha Shah, Rachana Rajpal, R.L. Tanna, Joydeep Ghosh, P.K. Chattopadhyay, R. Jha and Aditya Team "Multi-channel control circuit for real-time control of events in Aditya tokamak" Fusion Engineering and Design 112 (2016) 678-682

\section{Contribution of individual authors to the creation of a scientific article}

Minsha Shah, Hitesh Mandaliya have designed and developed trigger circuit of section 3 and also implemented programming in assembly language and LabVIEW of section 4.

Lavkesh Lachhvani, Manu Bajpai generated the requirement specifications and have organized and carried out the experiment as per the section 2

Rachana Rajpal supervised the development of trigger circuit.

\section{Sources of funding for research presented in a scientific article or scientific article itself}

\section{Institute for Plasma Research}

